Pacific Journal of Mathematics

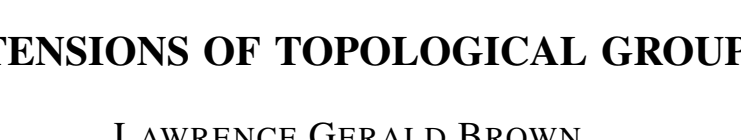




\section{EXTENSIONS OF TOPOLOGICAL GROUPS}

\section{LAWRENCE G. BROWN}

In this paper, we will be concerned with topological group extensions of a polonais group $A$ by a polonais group $G$. When $A$ is abelian, we will consider two cohomology groups: $H^{2}(G, A)$ and $H_{V}^{2}(G, A) . \quad H^{2}(G, A)$ is based on Borel cochains and was studied by Moore. $H_{V}^{2}(G, A)$ is obtained by identifying cochains which differ only on a first category set, an idea suggested to us by $D$. Wigner. We will show that each of the groups classifies the extensions and that the hypothesis that $A$ be abelian can be eliminated.

By a polonais group, we mean a separable metrizable topological group which is complete in its two-sided uniformity. The completeness requirement is equivalent to topological completeness (see [2], Exercise $Q(d)$, p. 212). If $E$ is a topological group having a polonais normal subgroup $A$ such that $E / A$ is polonais, then it is elementary to prove that $E$ must be polonais also.

If $A$ is abelian, then the cohomology groups are defined in terms of an a priori action of $G$ on $A$. If $A$ is non-abelian, then we will define sets (not groups) $H^{2}(G, A)$ without given action of $G$ on $A$. For brevity, we proceed directly to the general case.

Let $\mathscr{A}$ be the group of topological automorphisms of $A$. We will write ${ }^{\theta} a$ for the action of $\theta \in \mathscr{A}$ on $a \in A$ and $I_{a}$ for the inner automorphism $b \rightarrow a b a^{-1}$. Let $e$ denote the identity of any group. Then $H^{2}(G, A)$ is defined by means of cocycles $(\sigma, \rho)$ where:

$$
\sigma: G \times G \rightarrow A, \rho: G \rightarrow \mathscr{A},
$$

$\sigma$ is a Borel function on $G \times G$ and $(x, a) \rightarrow^{\rho(x)} a$ is a Borel function on $G \times A$.

$$
\begin{aligned}
\sigma(x, y) \cdot \sigma(x y, z) & ={ }^{\rho(x)} \sigma(y, z) \cdot \sigma(x, y z), \\
\rho(x) \cdot \rho(y) & =I_{\sigma(x, y)} \rho(x, y), \\
\sigma(x, e) & =\sigma(e, y)=e, \text { and } \\
\rho(e) & =e .
\end{aligned}
$$

$(\sigma, \rho)$ and $\left(\sigma^{\prime}, \rho^{\prime}\right)$ are identified in $H^{2}(G, A)$ if there is a Borel function $\lambda: G \rightarrow A$ such that:

$$
\begin{aligned}
\sigma^{\prime}(x, y) & =\lambda(x) \cdot \rho(x) \lambda(y) \cdot \sigma(x, y) \cdot \lambda(x y)^{-1}, \\
\rho^{\prime}(x) & =I_{\lambda(x)} \cdot \rho(x) .
\end{aligned}
$$

$H_{V}^{2}(G, A)$ can be defined by simply stipulating that (2) and (3) 
hold only on the complement of a first category set. However, for purposes of motivation, we prefer a slightly different description.

If $X$ is a complete metric space and $Y$ a metric space, then (following Wigner) by a virus from $X$ to $Y$, we mean an equivalence class of continuous functions $f: R \rightarrow Y$ where $R$ is a dense $G_{\delta}$ set in $X . f$ and $f^{\prime}$ are equivalent if they agree on $R \cap R^{\prime}$. (We could eliminate the equivalence relation by using maximal continuous extensions.) It is clear that if $Y$ is separable, the viruses amount to Borel functions modulo first category sets. The concept of virus will be used later. In the abelian case, it could be incorporated directly into the definition of $H_{V}^{2}(G, A)$.

$H_{V}^{2}(G, A)$ is defined by means of cocycles $(\sigma, \rho)$ where:

(1') $\sigma: R_{1} \rightarrow A, \rho: R_{2} \rightarrow \mathscr{A}$ where $R_{1}$ and $R_{2}$ are dense $G_{\delta}$ 's in $G \times G$ and $G . \quad \sigma$ is continuous on $R_{1}$, and $(x, a) \rightarrow^{\rho(x)} a$ is continuous on $R_{2} \times A$.

(2') $\sigma(x, y) \sigma(x y, z)={ }^{\rho(x)} \sigma(y, z) \sigma(x, y z)$ and $\rho(x) \rho(y)=I_{\sigma(x, y)} \rho(x y)$ whenever everything is defined. $(\sigma, \rho)$ and $\left(\sigma^{\prime}, \rho^{\prime}\right)$ are identified in $H_{V}^{2}(G, A)$ if there is a continuous function $\lambda: R \rightarrow A\left(R\right.$ a dense $G_{\delta}$ in $G)$ such that:

$\left(3^{\prime}\right)$ (3) holds whenever everything is defined. (In particular, $(\sigma, \rho)$ is identified with $\left(\sigma^{\prime}, \rho^{\prime}\right)$ if they agree off a first category set.)

We need two technical results:

Lemma 1 (Dixmier [1]). If $E$ is a polonais group, $A$ a closed normal subgroup, and $p: E \rightarrow E / A$ the projection, then there is a Borel function $f: E / A \rightarrow E$ such that $p(f(x))=x$ for all $x \in E / A$.

LEMma 2 (Wigner). If $E$ is a metrizable group complete in its two-sided uniformity, $A$ a closed normal subgroup, and $p: E \rightarrow E / A$ the projection, then there is a dense $G_{\delta}, R \subset E / A$ and a continuous function $f: R \rightarrow E$ such that $p(f(x))=x$ for all $x \in R$.

Proof. (One may note first that Lemma 2 follows from Lemma 1 in the polonais case.) Let $U_{1}, U_{2} \cdots$ be a fundamental system of symmetric neighborhoods of $e$ in $G$. Define recursively subsets $O_{n}$ of $G$ such that $O_{n}$ is maximal with respect to:

(4) $O_{n}$ is open; $O_{n} \subset O_{n-1}$; and if $x, y \in O_{n}$ and $p(x)=p(y)$, then $x^{-1} y, y x^{-1} \in U_{n} \cdot\left(O_{0}=E\right.$. $)$

It is not hard to see that $p\left(O_{n}\right)$ is dense in $E / A$ :

If $V \subset O_{n-1}$ is open, $V V^{-1}$ and $V^{-1} V \subset U_{n}$, and $p(V)$ is disjoint from $p\left(O_{n}\right)$, then $O_{n} \cup V$ satisfies (4). If $R=\cap p\left(O_{n}\right)$, then it is not hard to prove that $R$ is dense (i.e., that $E / A$ is second category in itself. Actually, $E / A$ is even complete in its two-sided uniformity.) For $x \in R$, let $y_{n} \in O_{n}$ be such that $p\left(y_{n}\right)=x$. Then $\left(y_{n}\right)$ is Cauchy 
and hence $y_{n} \rightarrow y$, for some $y \in G$. $y$ can easily be seen to be independent of the choices of $y_{n}$, and if we define $f(x)=y$, it is straightforward to prove $f$ is continuous.

Now we can define a map $\pi: \operatorname{Ext}(G, A) \rightarrow H^{2}(G, A) . \quad(\operatorname{Ext}(G, A)$ is the set of equivalence classes of topological group extensions of $A$ by $G$.) If $E$ is a given extension, let $f$ be as in Lemma 1 such that $f(e)=e$.

Define $(\sigma, \rho)$ by:

$$
f(x) \cdot f(y)=\sigma(x, y) f(x y) ;{ }^{\rho(x)} a=f(x) \cdot a \cdot f(x)^{-1} .
$$

It is easy to see that $\pi(E)=[(\sigma, \rho)]$ gives a well defined function $\pi$ where $[(\sigma, \rho)]$ denotes the class of $(\sigma, \rho)$.

There is a natural map $j: H^{2}(G, A) \rightarrow H_{V}^{2}(G, A)$. If $(\sigma, \rho)$ satisfies (1) and (2), then there are dense $G_{o}$ 's $R_{1} \subset G \times G$ and $R_{3} \subset G \times A$ such that the restriction of $\sigma$ to $R_{1}$ and the restriction of $(x, a) \rightarrow$ ${ }^{\rho(x)} a$ to $R_{3}$ are continuous.

LemmA 3. If $R_{2}=\left\{x \in G:\left\{a:(x, a) \in R_{3}\right\}\right.$ is a dense $G_{o}$ in $\left.A\right\}$, then $R_{2}$ is a dense $G_{o}$ in $G$ and $(x, a) \rightarrow{ }^{\rho(x)} a$ is continuous on $R_{2} \times A$.

Proof. That $R_{2}$ is a dense $G_{\delta}$ is clear. Now suppose $x_{n} \rightarrow x$ and $a_{n} \rightarrow a\left(x_{n}, x \in R_{2}\right)$. We can find $b \in A$ such that:

$$
\left(x_{n}, b\right) \in R_{3},(x, b) \in R_{3},\left(x_{n}, b^{-1} a_{n}\right) \in R_{3},\left(x, b^{-1} a\right) \in R_{3} .
$$

Then ${ }^{\rho\left(x_{n}\right)} a_{n}={ }^{\rho\left(x_{n}\right)} b \cdot{ }^{\rho\left(x_{n}\right)} b^{-1} a_{n} \rightarrow{ }^{\rho(x)} b \cdot{ }^{\rho(x)} b^{-1} a={ }^{\rho(x)} a$.

Now let $\bar{\sigma}$ and $\bar{\rho}$ be the restrictions of $\sigma$ and $\rho$ to $R_{1}$ and $R_{2}$, and define $j[(\sigma, \rho)]=[(\bar{\sigma}, \bar{\rho})]$. It should be clear that $j$ is a welldefined map on $H^{2}(G, A)$.

The map $j \pi: \operatorname{Ext}(G, A) \rightarrow H_{V}^{2}(G, A)$ can be described a little more simply. If $f$ and $R$ satisfy the conclusion of Lemma 2 , then we can use (5) to define $\sigma, \rho, R_{1}$ and $R_{2}$. Note that $R_{2}=R$ and $R_{1}=\widetilde{R}=$ $\{(x, y) \in G \times G: x, y, x y \in R\}$.

We can now state:

THEOREM. If $G$ and $A$ are polonais groups, then $\pi$ and $j \pi$ are bijections.

Proof. The main part of the proof is that $j \pi$ is surjective, and we prove that first. Let $\sigma, \rho, R_{1}$ and $R_{2}$ be given. We first reduce to the case where $R_{1}$ and $R_{2}$ are as in the previous paragraph. Let $R=\left\{x \in R_{2}:\left\{y:(x, y) \in R_{1}\right\}\right.$ is a dense $G_{\delta}$ in $\left.G\right\}$. Then for $(x, y) \in \widetilde{R}$, 
define:

(6) $\Sigma(x, y)(z)={ }^{\rho(x)} \sigma(y, z) \cdot \sigma(x, y z) \cdot \sigma(x y, z)^{-1}$ for all $z$ such that this makes sense.

Then $\Sigma$ induces a function from $\widetilde{R}$ into the set of viruses from $G$ to $A$. For $(x, y) \in \widetilde{R} \cap R_{1}$, it is easy to see from $\left(2^{\prime}\right)$ that $\Sigma(x, y)$ is the constant virus $\sigma(x, y)$. Since $\widetilde{R} \cap R_{1}$ is dense in $\widetilde{R}$ and $\Sigma$ is continuous, it is now clear that $\Sigma(x, y)$ is a constant virus, $\widetilde{\sigma}(x, y)$, for all $(x, y) \in \widetilde{R}$, and $\widetilde{\sigma}$ is continuous. We now replace $\sigma$ by $\widetilde{\sigma}$ (and $R_{2}$ by $R$ ) to obtain the desired situation. We will also assume that $e \notin R$.

We next extend $(\sigma, \rho)$ to a cocycle defined everywhere, following Moore [7]. To this end, let $V$ be the group of all viruses from $G$ to $A$. For $x \in R, a \in A$, define $\theta(x): V \rightarrow V$ and $J_{a}: V \rightarrow V$ by:

$$
\begin{aligned}
{[\theta(x) g](y) } & =\sigma(y, x) g(y x) \text { and } \\
\left(J_{a} g\right)(x) & ={ }^{\rho(x)} a \cdot g(x) \text { for } g \in V .
\end{aligned}
$$

It is a straightforward computation to verify:

$$
\begin{aligned}
& \theta(x) \cdot \theta(y)=J_{\sigma(x y)} \cdot \theta(x y),(x, y, x y \in R), J_{a b}=J_{a} \cdot J_{b}, \text { and } \\
& \theta(x)^{-1} \text { exists and } \theta(x) J_{a} \theta(x)^{-1}=J_{\rho(x)_{a}} .
\end{aligned}
$$

Now let $E^{\prime}$ be the group generated by $J(A)$ and $\theta(R)$. We can see that if $A$ is identified with $J(A)$, then $A$ is normal in $E^{\prime}$, and each element of $E^{\prime}$ induces a continuous automorphism of $A$. Let $p: E^{\prime} \rightarrow$ $E^{\prime} / A$ be the projection. Clearly, for $(x, y) \in \widetilde{R}, p \theta(x y)=p \theta(x) \cdot p \theta(y)$. It is now easy to see that $p \theta$ extends to a homomorphism $\bar{\theta}: G \rightarrow$ $E^{\prime} / A$. Let $f: G \rightarrow E^{\prime}$ be an extension of $\theta$ such that $f(e)=e$ and $p f=\bar{\theta}$. Then we can extend $(\sigma, \rho)$ by:

$$
\begin{gathered}
f(x) \cdot f(y)=J_{\sigma(x y)} \cdot f(x y), \text { and } \\
J_{\rho(x)_{a}}=f(x) \cdot J_{a} \cdot f(x)^{-1} .
\end{gathered}
$$

It is clear that the extended $(\sigma, \rho)$ satisfies the cocycle relations (2) (though not (1)).

We will later have occasion to use a uniqueness result for the extended $(\sigma, \rho)$. Thus let $\left(\sigma^{\prime}, \rho^{\prime}\right)$ satisfy (2) everywhere, suppose $\sigma^{\prime}$ agrees with $\sigma$ on a dense $G_{\dot{\delta}}, R_{1}$, and $\rho^{\prime}$ agrees with $\rho$ on a dense $G_{\dot{\delta}}, R_{2}$. Define $R^{\prime}=\left\{y \in R \cap R_{2}:\left\{x:(x, y) \in R_{1}\right\}\right.$ is a dense $G_{\delta}$ in $\left.G\right\}$. We can see that for any $v \in G$, there is a dense $G_{\hat{\delta}}, R_{v}$, such that $\sigma^{\prime}(u, v)$ is continuous in $u$ on $R_{v}$. This follows from the following consequence of $(2)$ :

$$
\sigma^{\prime}(u, y z)=\rho^{\prime}(u) \sigma^{\prime}(y, z)^{-1} \cdot \sigma^{\prime}(u, y) \cdot \sigma^{\prime}(u y, z)
$$

where we choose $y, z \in R^{\prime}$ such that $y z=v$. 
Thus we can use (7) to define $\theta^{\prime}$ on all of $G$, agreeing with $\theta$ on $R^{\prime}$, and $\theta^{\prime}$ will satisfy (8) (with $\left(\sigma^{\prime}, \rho^{\prime}\right)$ instead of $(\sigma, \rho)$ ). If we define $\lambda$ by $\theta^{\prime}(x)=\lambda(x) f(x)$, then we see that (3) is satisfied and $\lambda$ vanishes on $R^{\prime}$. This is the desired uniqueness result.

Using the extended $(\sigma, \rho)$, we can now construct a (non-topological) extension $E$ of $A$ by $G$ and a function $f: G \rightarrow E$ such that if $p: E \rightarrow G$ is the projection, then $p f(x)=x, f(e)=e$, and (5) is satisfied. We must topologize $E$, and we first define sequential convergence to $e$. If $\alpha_{n} \in E$, we say that $\alpha_{n} \rightarrow e$ if there exists $\beta=b \cdot f(y)(y \in R)$ such that if $\beta \alpha_{n}=b_{n} \cdot f\left(y_{n}\right)$, then $y_{n} \in R, b_{n} \rightarrow b$, and $y_{n} \rightarrow y$. If $\alpha_{n}=a_{n} \cdot f\left(x_{n}\right)$, then it is readily seen that this condition is equivalent to:

$$
y x_{n} \in R, x_{n} \rightarrow e \text {, and }{ }^{\rho(y)} a_{n} \cdot \sigma\left(y, x_{n}\right) \rightarrow e .
$$

The condition is thus independent of $b$, and we now show it is independent of $y$ (subject to $y, y x_{n} \in R$ ). We need:

Lemma 4. (a) For $x \in G, \sigma(x, y)$ is continuous in $y$ on $R_{x}=$ $\{y: y, x y \in R\}$.

(b) $\sigma(x, y)$ is continuous in $x$ on $R_{y}=\{x: x, x y \in R\}$.

Proof. (a) Let $y_{n} \rightarrow y$ in $R_{x}$. Consider:

$$
\sigma\left(x, y_{n}\right)=\sigma(v, w)^{-1} \cdot \rho(v) \sigma\left(w, y_{n}\right) \cdot \sigma\left(v, w y_{n}\right)
$$

where $v=x w^{-1}$ and $\quad w, x w^{-1}, w y_{n}, w y \in R$.

Then from the continuity of $\sigma$ on $\widetilde{R}$, we see that $\sigma\left(x, y_{n}\right) \rightarrow \sigma(x, y)$.

(b) is proved similarly.

Now if $y=u y^{\prime}$ where $y, y x_{n}, y^{\prime}, y^{\prime} x_{n} \in R$, we find:

$$
{ }^{\rho(y)} a_{n} \cdot \sigma\left(y, x_{n}\right)=\sigma\left(u, y^{\prime}\right)^{-1 \rho(u)}\left[\rho^{\left(y^{\prime}\right)} a_{n} \cdot \sigma\left(y^{\prime}, x_{n}\right)\right] \cdot \sigma\left(u, y^{\prime} x_{n}\right) .
$$

Using Lemma 4(a), we see that (11) is satisfied for $y$ if it is for $y^{\prime}$.

Lemma 5. If $\alpha_{n} \rightarrow e$, then $\beta \alpha_{n} \beta^{-1} \rightarrow e$ for any $\beta \in E$.

Proof. First, let $\beta=b \in A$ where $\alpha_{n}=a_{n} f\left(x_{n}\right)$. If $y, y x_{n} \in R$ and $f(y) \beta \alpha_{n} \beta^{-1}=c_{n} \cdot f\left(x_{n}\right)$, then:

$$
c_{n}={ }^{\rho(y)} b \cdot\left[{ }^{\rho(y)} a_{n} \cdot \sigma\left(y, x_{n}\right)\right] \cdot \rho^{\left(y x_{n}\right)} b^{-1} .
$$

Hence (11) is satisfied for $\beta \alpha_{n} \beta^{-1}$ if satisfied for $\alpha_{n}$. Now let $\beta=f(z)$. Then if $y, y z x_{n} z^{-1}, y z, y z x_{n} \in R$, and $f(y) \beta \alpha_{n} \beta^{-1}=d_{n} f\left(y z x_{n} z^{-1}\right)$,

$$
d_{n}=\sigma(y, z) \cdot\left[\rho^{(y z)} a_{n} \cdot \sigma\left(y z, x_{n}\right)\right] \cdot \sigma\left(y z x_{n} z^{-1}, z\right)^{-1} \cdot
$$

Hence (by Lemma 4 (b)), (11) is satisfied for $\beta \alpha_{n} \beta^{-1}$ if satisfied for $\alpha_{n}$. 
From this lemma, it is easy to see that the definition of $\alpha_{n} \rightarrow e$ could equivalently have been given in terms of $\alpha_{n} \beta$ instead of $\beta \alpha_{n}$. The criterion would then have been:

$\left(11^{\prime}\right)$ If $w, x_{n} w \in R$, then $a_{n} \cdot \sigma\left(x_{n}, w\right) \rightarrow e$ and $x_{n} \rightarrow e . \quad\left(\alpha_{n}=a_{n} \cdot f\left(x_{n}\right)\right)$.

LEMma 6. If $\alpha_{n} \rightarrow e$ and $\beta_{n} \rightarrow e$, then $\alpha_{n} \cdot \beta_{n} \rightarrow e$.

Proof. Let $\alpha_{n}=a_{n} \cdot f\left(x_{n}\right), \beta_{n}=b_{n} \cdot f\left(y_{n}\right)$. First, choose $z$ such that $z, z x_{n}$, and $z x_{n} y_{n} \in R$. Then choose $w$ such that $w, y_{n} w$, and $z x_{n} y_{n} w \in R$. Then if $f(z) \cdot \alpha_{n} \cdot \beta_{n}=c_{n} f\left(z x_{n} y_{n}\right)$, we find:

$$
c_{n}=\left[{ }^{\rho(z)} a_{n} \cdot \sigma\left(z, x_{n}\right)\right] \cdot{ }^{\rho\left(z x_{n}\right)}\left[b_{n} \cdot \sigma\left(y_{n}, w\right)\right] \cdot \sigma\left(z x_{n}, y_{n} w\right) \cdot \sigma\left(z x_{n}, y_{n}, w\right)^{-1} \cdot
$$

Hence (11), for $\alpha_{n} \beta_{n}$ follows from (11) for $\alpha_{n}$ and (11') for $\beta_{n}$.

LEMMA 7. If $\alpha_{n} \rightarrow e, \alpha_{n}^{-1} \rightarrow e$.

Proof. Let $\alpha_{n}=a_{n} \cdot f\left(x_{n}\right)$. Choose $z$ such that $z, z x_{n}^{-1} \in R$. Then choose $w$ such that $w, z w, x_{n} w \in R$. Then if $f(z) \cdot \alpha_{n}^{-1}=c_{n} f\left(z x_{n}^{-1}\right)$, we find

$$
c_{n}^{-1}={ }^{\rho\left(z x_{n}^{-1}\right)}\left[a_{n} \cdot \sigma\left(x_{n}, w\right)\right] \cdot \sigma\left(z x_{n}^{-1}, x_{n} w\right) \cdot \sigma(z, w)^{-1} .
$$

Thus (11), for $\alpha_{n}^{-1}$ follows from (11') for $\alpha_{n}$.

We now must show that there is a metrizable group-topology on $E$ such that convergence as defined above is convergence in the topology. To do this, it is sufficient to find a sequence $W_{m}$ such that $e \in W_{m} \subset E$, and $\alpha_{n} \rightarrow e$ as defined above if and only if $\alpha_{n}$ is eventually in $W_{m}$, for each $m$. Let $U_{m}$ be a fundamental system of neighborhoods of $e$ in $A$ and $V_{m}$ a fundamental system of neighborhoods relative to $R$ of $u_{0} \in R$. Then we define:

$$
W_{m}=\left[U_{m} f\left(V_{m}\right)\right]^{-1} \cdot U_{m} f\left(V_{m}\right) .
$$

Suppose that $\alpha_{n}$ is eventually in each $W_{m}$. Then $\alpha_{n}=\beta_{n}^{-1} \gamma_{n}$ where $\beta_{n}, \gamma_{n}$ are eventually in each $U_{m} f\left(V_{m}\right)$. Hence $f\left(u_{0}\right)^{-1} \beta_{n} \rightarrow e$ and $f\left(u_{0}\right)^{-1} \gamma_{n} \rightarrow e$. Thus $\alpha_{n}=\left[f\left(u_{0}\right)^{-1} \beta_{n}\right]^{-1} \cdot\left[f\left(u_{0}\right)^{-1} \gamma_{n}\right] \rightarrow e$. Now assume $\alpha_{n}=$ $a_{n} f\left(x_{n}\right) \rightarrow e$. Since $V_{m}^{-1} V_{m}$ is a neighborhood of $e$ in $G$, we can find $y_{n}, z_{n} \in R$ such that $y_{n} \rightarrow u_{0}, z_{n} \rightarrow u_{0}$ and $y_{n}^{-1} z_{n}=x_{n}$. Now define $\beta_{n}=f\left(y_{n}\right)$ and $\gamma_{n}=c_{n} f\left(z_{n}\right)$ where $c_{n}$ is chosen such that $\beta_{n}^{-1} \gamma_{n}=\alpha_{n}$. Then calculation shows:

$$
c_{n}={ }^{\rho\left(y_{n}\right)}\left[a_{n} \cdot \sigma\left(x_{n}, w\right)\right] \cdot \sigma\left(y_{n}, x_{n} w\right) \cdot \sigma\left(z_{n}, w\right)^{-1}
$$

where we assume $w$ chosen so that $w, x_{n} w, z_{n} w$ and $u_{0} w \in R$. Then (11') shows that $c_{n} \rightarrow e$. Hence $\beta_{n}, \gamma_{n}$ are eventually in each $U_{m} f\left(V_{m}\right)$, as 
desired.

Now that we have a metrizable topology on $E$, it is easy to see that the subspace topology on $A$ is the correct one. It is also not hard to see that the quotient topology on $G$ is the correct one. Indeed, all we must show is that if $x_{n} \rightarrow e$ in $G$, then we can find $\alpha_{n} \rightarrow e$ in $E$ such that $p\left(\alpha_{n}\right)=x_{n}$. To do this, choose $y_{n}$ and $z_{n}$ as in the preceding paragraph. Then $\alpha_{n}=f\left(y_{n}\right)^{-1} f\left(z_{n}\right)$. From the fact that $A$ and $G$ are complete and separable, it now follows easily that $E$ is complete and separable and hence polonais. It is clear that $f$ is continuous on $R$. Hence $j \pi(E)=[(\sigma, \rho)]$, and we have proved that $j \pi$ is surjective.

To show that $j \pi$ is $1-1$, assume $j \pi(E)=j \pi\left(E^{\prime}\right)$. Then we can find a dense $G_{\delta} R \subset G$ and continuous $f: R \rightarrow E, f^{\prime}: R \rightarrow E^{\prime}$ such that $p f(x)=x=p^{\prime} f^{\prime}(x)$, and $(\sigma, \rho)=\left(\sigma^{\prime}, \rho^{\prime}\right)$ where $\sigma, \rho, \sigma^{\prime}$, and $\rho^{\prime}$ are defined by (5). Then we can define a function $\varphi: p^{-1}(R) \rightarrow p^{-1}(R)$ by:

$$
\varphi(a \cdot f(x))=a \cdot f^{\prime}(x),
$$

and clearly $\varphi(\alpha \beta)=\varphi(\alpha) \cdot \varphi(\beta)$ for $\alpha, \beta, \alpha \beta \in p^{-1}(R)$. From this, it is not hard to prove that $\varphi$ can be extended to a homomorphism of $E$ onto $E^{\prime}$ and that this homomorphism is a topological isomorphism.

It is now clear that $\pi$ is $1-1$. To show that $\pi$ is surjective, let $(\sigma, \rho)$ satisfy (1) and (2). Let $E$ be such that $j \pi(E)=j[(\sigma, \rho)]$. Then we can find a Borel function $f: G \rightarrow E$ such that $p f(x)=x$, $f(e)=e$, and if $\left(\sigma^{\prime}, \rho^{\prime}\right)$ is defined by (5) (with $\left(\sigma^{\prime}, \rho^{\prime}\right)$ substituted for $(\sigma, \rho))$, then $(\sigma, \rho)$ agrees with $\left(\sigma^{\prime}, \rho^{\prime}\right)$ except on a first category set. Now the uniqueness result proved above shows that there is a function $\lambda: G \rightarrow A$, which vanishes on a dense $G_{\hat{o}}, R^{\prime}$, such that (3) holds. We must show that $\lambda$ is Borel. (3) implies:

$$
\lambda(x y)=\sigma^{\prime}(x, y)^{-1} \cdot \lambda(x) \cdot{ }^{\rho(x)} \lambda(y) \cdot \sigma(x, y)=\sigma^{\prime}(x, y)^{-1} \cdot \sigma(x, y),
$$

for $(x, y) \in R^{\prime} \times R^{\prime}$. Thus if $m: R^{\prime} \times R^{\prime} \rightarrow G$ is the group operation, then $\lambda \circ m$ is Borel. Since $m$ is surjective, it follows from well-known results on Borel sets (see Kuratowski [3] or the first few pages of Mackey [4]) that $\lambda$ is Borel. The theorem is now proved.

We make two final remarks:

1. If $A$ is abelian and we are given an action of $G$ on $A$, then it is easy to see that the bijections $\pi, j$, and $j \pi$ preserve the group operations (where the group operation on $\operatorname{Ext}(G, A)$ is the usual Baer product).

2. The hypothesis that $A$ be separable can be dropped by considering $A$ as a direct limit of closed separable subgroups ( $G$-subgroups 


\section{in the abelian case).}

\section{REFERENCES}

1. J. Dixmier, Dual et quasi-dual d'une algebre de Banach involutive, Trans. Amer. Math. Soc., 104 (1962), 278-283.

2. J. Kelley, General Topology, Van Nostrand, Princeton, 1955.

3. K. Kuratowski, Topology Vol. I (translated from the French by J. Jawarowski), Academic Press, New York, 1966.

4. G. Mackey, Borel structures in groups and their duals, Trans. Amer. Math. Soc., 85 (1957), 134-165,

5. C. Moore, Extensions and low dimensional cohomology theory of locally compact groups I, Trans. Amer. Math. Soc., 113 (1964), 40-63.

6. - Extensions and low dimensional cohomology theory of locally compact groups II, Trans. Amer. Math. Soc., 113 (1964), 64-86.

7. - Group extensions of p-adic and adelic linear groups, I.H.E.S. Publ. Math., 35 (1968), 5-70.

Received November 2, 1970.

STANFORD UNIVERSITY 


\section{PACIFIC JOURNAL OF MATHEMATICS}

\section{EDITORS}

H. SAMELSON

Stanford University

Stanford, California 94305

C. R. HobBY

University of Washington

Seattle, Washington 98105
J. DugundjI

Department of Mathematics

University of Southern California

Los Angeles, California 90007

RICHARD ARENS

University of California

Los Angeles, California 90024

\section{ASSOCIATE EDITORS}

E. F. BECKENBACH

B. H. NeUMaNN

F. WOLF

K. YoshidA

\section{SUPPORTING INSTITUTIONS}

UNIVERSITY OF BRITISH COLUMBIA

CALIFORNIA INSTITUTE OF TECHNOLOGY

UNIVERSITY OF CALIFORNIA

MONTANA STATE UNIVERSITY

UNIVERSITY OF NEVADA

NEW MEXICO STATE UNIVERSITY

OREGON STATE UNIVERSITY

UNIVERSITY OF OREGON

OSAKA UNIVERSITY
UNIVERSITY OF SOUTHERN CALIFORNIA STANFORD UNIVERSITY

UNIVERSITY OF TOKYO

UNIVERSITY OF UTAH

WASHINGTON STATE UNIVERSITY UNIVERSITY OF WASHINGTON

$\stackrel{*}{*} \stackrel{*}{*} \stackrel{*}{*}$ AMERICAN MATHEMATICAL SOCIETY
NAVAL WEAPONS CENTER 


\section{Pacific Journal of Mathematics}

Vol. 39 , No. 1

May, 1971

Charles A. Akemann, A Gelfand representation theory for $C^{*}$-algebras ....

Sorrell Berman, Spectral theory for a first-order symmetric system of

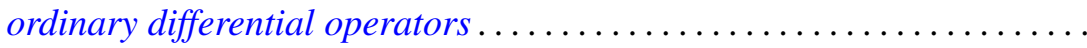

Robert L. Bernhardt, III, On splitting in hereditary torsion theories ........

J. L. Brenner, Geršgorin theorems, regularity theorems, and bounds for determinants of partitioned matrices. II. Some determinantal identities ..........................................

Robert Morgan Brooks, On representing $F^{*}$-algebras .............. 51

Lawrence Gerald Brown, Extensions of topological groups........... 71

Arnold Barry Calica, Reversible homeomorphisms of the real line ........ 79

J. T. Chambers and Shinnosuke Oharu, Semi-groups of local Lipschitzians in

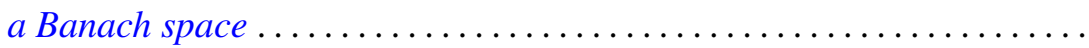

Thomas J. Cheatham, Finite dimensional torsion free rings .............

Byron C. Drachman and David Paul Kraines, A duality between

transpotence elements and Massey products ...................

Richard D. Duncan, Integral representation of excessive functions of a

Markov process ......................................

George A. Elliott, An extension of some results of Takesaki in the reduction

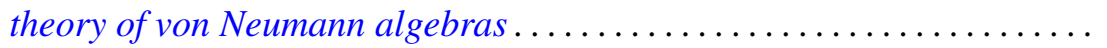

Peter C. Fishburn and Joel Spencer, Directed graphs as unions of partial

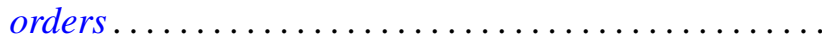

Howard Edwin Gorman, Zero divisors in differential rings ...

Maurice Heins, A note on the Löwner differential equations...

Louis Melvin Herman, Semi-orthogonality in Rickart rings. .

David Jacobson and Kenneth S. Williams, On the solution of linear G.C.D.

equations

Michael Joseph Kallaher, On rank 3 projective planes ... . .

Donald Paul Minassian, On solvable $O^{*}$-groups ...........

Nils Øvrelid, Generators of the maximal ideals of $A(\bar{D})$

Mohan S. Putcha and Julian Weissglass, A semilattice decomposition into

semigroups having at most one idempotent ............

Robert Raphael, Rings of quotients and $\pi$-regularity ....

J. A. Siddiqi, Infinite matrices summing every almost periodic sequence. .

Raymond Earl Smithson, Uniform convergence for multifunctions ...

Thomas Paul Whaley, Mulitplicity type and congruence relations in

universal algebras...

Roger Allen Wiegand, Globalization theorems for locally finitely generated modules... 